Article

\title{
Correcting Errors in Image Encryption Based on DNA Coding
}

\author{
Bin Wang ${ }^{1, *(1)}$, Yingjie Xie ${ }^{2}$, Shihua Zhou ${ }^{1}$, Xuedong Zheng ${ }^{1}$ and Changjun Zhou ${ }^{3, *}$ \\ 1 Key Laboratory of Advanced Design and Intelligent Computing, Dalian University, Ministry of Education, \\ Dalian 116622, China; shihuajo@gmail.com (S.Z.); xuedongzheng@163.com (X.Z.) \\ 2 Applied Technology College, Dalian Ocean University, Dalian 116300, China; yingjieying@163.com \\ 3 College of Mathematics, Physics and Information Engineering, Zhejiang Normal University, \\ Jinhua 321004, China \\ * Correspondence: wangbinpaper@gmail.com (B.W.); zhou-chang231@163.com (C.Z.); \\ Tel.: +86-0411-8740-2106 (B.W. \& C.Z.)
}

Received: 25 June 2018; Accepted: 24 July 2018; Published: 27 July 2018

\begin{abstract}
As a primary method, image encryption is widely used to protect the security of image information. In recent years, image encryption pays attention to the combination with DNA computing. In this work, we propose a novel method to correct errors in image encryption, which results from the uncertainty of DNA computing. DNA coding is the key step for DNA computing that could decrease the similarity of DNA sequences in DNA computing as well as correct errors from the process of image encryption and decryption. The experimental results show our method could be used to correct errors in image encryption based on DNA coding.
\end{abstract}

Keywords: image encryption; chaotic map; DNA coding; Hamming distance

\section{Introduction}

With wide usage of multimedia technologies and excessive spread of internet, the awareness of protecting information, especially image information, is heightened day by day. As we known, encrypting technology can usually be used to protect the security of image information. In image encryption, chaotic maps are usually employed to encrypt image, because they have the features of ergodicity, sensitivity to initial conditions, control parameters and so on [1-7]. Chen et al. proposed a novel 3D cat maps to design a real-time secure symmetric encryption scheme [1]. Lian et al. first analyzed the parameter sensitivity of standard map and proposed an improved standard map to encrypt image [3]. Wong et al. proposed a fast algorithm of image encryption, where the overall encryption time was reduced as fewer rounds were required [2]. Zhang et al. proposed a new image encryption algorithm based on the spatiotemporal chaos of the mixed linear-nonlinear coupled map lattices [7]. Wang et al. combined genetic recombination with hyper-chaotic system to design a novel image encryption and experiment results proved that the proposed algorithm was effective for image encryption [8]. Zhang et al. analyzed different kinds of permutation algorithms and proposed a new cryptosystem to address these drawbacks [5]. In the recent past, although these methods have made some progress, they lack the capability of parallel computing.

Inspired by the biological character of DNA sequences, such as parallel computing, low-energy and so on, DNA computing and DNA coding are widely used to encrypt image [6,9-17]. Zhang et al. combined DNA sequence addition operation with chaotic map to design a novel image encryption scheme [9]. The experimental results shown that the proposed scheme could achieve good encryption and resist some kind attacks. In Ref. [10], the authors transformed DNA sequences into its base pair for random times to confuse the pixels, generate the new keys according to the plain image and the 
common keys. Wei et al. further utilized DNA sequence addition operation and Chen's hyper-chaotic map to encrypt a color image [11]. Due to some disadvantages in One-Time-Pad (OTP) algorithm, the author used logistic chaotic map as an input of OTP algorithm and proposed an interesting encryption algorithm based on a chaotic selection between original message DNA strands and OTP DNA strands [12]. In Ref. [13], the authors used genetic algorithm to determine the best masks, which result from DNA and logistic map functions. Ozkaynak et al. broke a previous cryptosystem and proposed an improved image encryption algorithm [14]. Rehman et al. utilized whole set of DNA complementary rules dynamically and employed DNA addition operation to encrypt image [15]. Song and Qiao proposed a novel image encryption scheme based on DNA encoding and spatiotemporal chaos, which was of high key sensitivity and large key space [16]. In Ref. [17], DNA coding combined with an improved 1D chaotic systems to design image encryption. Kulsoom et al. employed an entire set of DNA complementary rules along with 1D chaotic maps to design an image encryption algorithm [6]. Wang et al. proposed a new chaotic image encryption scheme based on Josephus traversing and mixed chaotic map [18]. Parvaz and Zarebnia defined a combination chaotic system and studied its properties [19].

DNA computing was addressed to solve the seven-point Hamiltonian path problem by Adleman in 1994 [20]. Along with the development of research, there are a large number of applications about DNA computing, such as DNA logic gates [21], neural network [22], cryptography [4], data storage [23], image watermarking [24] and so on. Hybridization reaction is the key operation for DNA sequences and influences the reliability of DNA computing. However, the false hybridization is unavoidable because of the limit of biological technology, result from false positive and false negative. The lack of similarity between DNA sequences could result in false positive and generating hybridization reaction between two unmatched DNA sequences. The mistake in the biochemical operation result in false negative in which two matched DNA sequences did not hybridize each other [25]. Chai et al. encoded plain image by DNA matrix and permuted the image with a new wave-based permutation scheme [26]. In Ref. [27], DNA sequence operation combining with one-way coupled-map lattices was to structure a robust and lossless color image encryption algorithm and the three gray-level components of plain-image were converted into three DNA matrices and performed XOR operation twice. Designing DNA coding could obtain high quality DNA sequences which satisfy some constraints, such as Hamming distances, GC content and so on, to decrease the similarity between DNA sequences [28,29]. Inspired by Hybridization reaction is the kernel for DNA computing and influences the reliability of DNA computing. However, the false hybridization is unavoidable because of the limit of biological technology, result from false positive and false negative. The lack of similarity between DNA sequences could result in false positive and generating hybridization reaction between two unmatched DNA sequences. The mistake in the biochemical operation result in false negative in which two matched DNA sequences did not hybridize each other [25]. Designing DNA coding could obtain high quality DNA sequences which satisfy some constraints, such as Hamming distance, GC content and so on, to decrease the similarity between DNA sequences $[28,29]$. Inspired by communication theory, Hamming code can be used to correct errors. For example, $d$ is the Hamming distance between two strings and then the bits of correcting errors are equal to $\left\lfloor\frac{d-1}{2}\right\rfloor$. So, in this paper, we introduce Hamming distance to decrease the similarity between DNA sequences as well as correct errors from hybridization reaction. Furthermore, to improve the accuracy of DNA computing, the constraints of DNA coding are used to decrease the generation of false positive. Finally, the experimental results show that the number of pixels change rate (NPCR) has achieved $99.57 \%$ and the unified average changing intensity (UACI) has achieved $32.38 \%$. The proposed method could effectively correct the encrypted image contained 1000 errors and improve the accuracy of hybridization reaction. 


\section{Methods}

\subsection{DNA Coding}

Hamming distance is widely used to design DNA coding. It is the number of positions at which the corresponding symbols are different when two strings have the equal length [30]. In the alphabet $\Sigma=\{\mathrm{A}, \mathrm{C}, \mathrm{G}, \mathrm{T}\}$, there exists a set $\mathrm{S}$ with length $\mathrm{n}$ and size of $|S|=4^{n}$. A subset $C \subseteq S$ and let $u, v$ any two codes in the $C$ satisfy [31]:

$$
\tau(u, v) \geq d
$$

$d$ is a positive integer, $\tau$ is the constraint criteria (or criterion) for designing DNA coding. In this paper, $\tau$ is denoted as the Hamming distance.

\subsubsection{Sequences-Sequence Hamming Distance (SS)}

Sequences-sequence Hamming Distance [31]: for the DNA sequences $u, v$ with given length $n$ (written from the $5^{\prime}$ to the $3^{\prime}$ end), the Hamming distance between $u$ and $v$ is denoted as $H(u, v)$. The minimal $H\left(u_{i}, v_{j}\right)$ in all DNA sequences is denoted as $S S\left(u_{i}\right)$ and it should not be less than parameter $d$,

$$
S S\left(u_{i}\right)=\min _{1 \leq j \leq n, j \neq i}\left\{H\left(u_{i}, v_{j}\right)\right\} \geq d
$$

\subsubsection{Sequences-Complementarity Hamming Distance (SC)}

Sequences-complementarity Hamming Distance [31]: for the DNA sequences $u, v$ with given length $n$ (written from the $5^{\prime}$ to the $3^{\prime}$ end), $H\left(u, v^{C}\right)$ denotes the Hamming distance between $u$ and $v^{C}$, where $v^{C}$ is the complementary sequence of $v$. For example, $v=$ ACTG, then $v^{C}=$ CAGT. The minimal $H\left(u_{i}, v_{j}^{C}\right)$ in all DNA sequences is denoted as $S S\left(u_{i}\right)$ and it should not be less than parameter $d$,

$$
S C\left(u_{i}\right)=\min _{1 \leq j \leq n, j \neq i}\left\{H\left(u_{i}, v_{j}^{C}\right)\right\} \geq d
$$

\subsubsection{GC Content}

In order to approximate the thermodynamic properties of DNA sequences, GC content constraint is used to combine with distance constraint, such as Hamming distance. The percentage of $G$ or $C$ bases within each DNA is denoted as GC content. In this paper, GC content is equal to $50 \%$. The GC content is denoted as follows:

$$
\text { GC_content }=N u m_{-} g c / n \times 100 \%
$$

\subsubsection{DNA Coding Rule}

Adenine (A), Cytosine (C), Guanine (G) and Thymine (T) are the four elements that make up the whole DNA sequence. When paring, the principle of complementary base pairing is observed, namely A with $\mathrm{T}$ and $\mathrm{C}$ with $\mathrm{G}$ [32]. There is a complementary relationship between 0 and 1 in the binary bit. Similarly, there is a complementary relationship between 01 and 10 as well as 00 and 11 . In the previous works, the authors converted binary message to DNA sequences based on the DNA coding rule in Table $1[6,9,13-15,17,23,33,34]$. There are eight DNA coding methods to convert binary message to DNA sequences $[9,24]$. For example, the pixel 65 is firstly transformed into binary bit 01000001 and then 01000001 transformed into DNA sequence ACCA for the first rule. 
Table 1. DNA coding list $[9,24]$.

\begin{tabular}{ccccccccc}
\hline & $\mathbf{1}$ & $\mathbf{2}$ & $\mathbf{3}$ & $\mathbf{4}$ & $\mathbf{5}$ & $\mathbf{6}$ & $\mathbf{7}$ & $\mathbf{8}$ \\
\hline $\mathrm{A}$ & 01 & 01 & 00 & 00 & 10 & 10 & 11 & 11 \\
$\mathrm{~T}$ & 10 & 10 & 11 & 11 & 01 & 01 & 00 & 00 \\
$\mathrm{C}$ & 00 & 11 & 01 & 10 & 00 & 11 & 01 & 10 \\
$\mathrm{G}$ & 11 & 00 & 10 & 01 & 11 & 00 & 10 & 01 \\
\hline
\end{tabular}

From the Table 1, the information only is simple transformed between binary and DNA sequence. It does not consider the characters of DNA sequences, especially specific hybridization. So, in this paper, we designed DNA coding that satisfied three constraints above to encrypt image and use this DNA coding to correct the errors.

\subsection{New DNA Coding Rule for Correcting Errors}

As shown above, the DNA sequences used to encode pixels and chaotic orbits should satisfy these constraints to decrease the similarity between DNA sequences and correct the errors. In our previous work, we proposed a dynamic genetic algorithm to design DNA sequence sets which satisfy the combinational constraints [35]. DNA sequence set denotes that any pair of DNA sequences in this set satisfies the combinational constraints. In this paper, we use the DNA coding $A_{4}^{S S+S C+G C}(8,3)=336$, namely the length is equal to 8 and Hamming distance is equal to 3 . We randomly select 256 elements from this set to encode the pixels between 0 and 255. In this paper, we denote these 256 DNA sequences as DNA coding rule. Table 2 lists the first 50 DNA coding rule to encrypt image. The whole $A_{4}^{S S+S C+G C}(8,3)$ and DNA coding rule are shown in the Supplement.

Table 2. The first 50 DNA coding rule.

\begin{tabular}{cccccccccc}
\hline Pixel & $\begin{array}{c}\text { DNA } \\
\text { Coding }\end{array}$ & Pixel & $\begin{array}{c}\text { DNA } \\
\text { Coding }\end{array}$ & Pixel & $\begin{array}{c}\text { DNA } \\
\text { Coding }\end{array}$ & Pixel & $\begin{array}{c}\text { DNA } \\
\text { Coding }\end{array}$ & $\begin{array}{c}\text { Pixel } \\
\text { Coding }\end{array}$ \\
\hline 0 & ATCATGCC & 1 & CTCGATCA & 2 & GCTCTTCT & 3 & AGTGGGAT & 4 & ACTCTCTG \\
5 & AATCTGCG & 6 & ACTCACGT & 7 & CTTCCAAC & 8 & GCTTCTAG & 9 & TAGGAGGT \\
10 & GATCGACT & 11 & TAACGCTG & 12 & TAAGCGGA & 13 & CTGTGATC & 14 & CCCTAATC \\
15 & TGGAAGGA & 16 & TACTACCG & 17 & CTTATGGG & 18 & TCAGCAAG & 19 & CGACTTCT \\
20 & AGTGTCGA & 21 & TGCGATTC & 22 & CAACGACA & 23 & GATCTGTC & 24 & GCCAACTA \\
25 & ATGAGGGA & 26 & TAGAACGG & 27 & CCGTAACA & 28 & TAGACTGC & 29 & GCTGGATT \\
30 & GTGAGTCA & 31 & TCATGGAC & 32 & ACCACTAC & 33 & TCCTAAGG & 34 & GGCTAAAG \\
35 & CCAACTGA & 36 & TCGTCTTG & 37 & TTGGGAAC & 38 & AATAGCCC & 39 & CTGTCGAA \\
40 & CCCCATAT & 41 & AACCTCTC & 42 & GGTTTACG & 43 & GCAGAAGA & 44 & TAGAGGAG \\
45 & GAAAGGGA & 46 & ATCGACGA & 47 & GCAAGTAC & 48 & TCAGACAC & 49 & CTTGGTTG \\
\hline
\end{tabular}

\subsection{Process of Encrypting and Decrypting Image Based on DNA Coding}

\subsubsection{Encrypting Image}

Recently, there are some works on cryptanalysis of encrypting schemes based on chaotic map and DNA coding [36-38]. In this paper, in order to improving the security of our encrypting scheme, two logistic maps with different parameters and initial values are chosen to generate pseudorandom sequence. The different parameters and initial values for the Equation (5) are denoted as $\mu_{1}, \mu_{2}, x_{1}^{1}(0)$ and $x_{1}^{2}(0)$, respectively, where $\mu_{1}, \mu_{2} \in[3.9,4]$ and $x_{1}^{1}(0), x_{1}^{2}(0) \in(0,1)$.

$$
x_{i+1}=\mu x_{i}\left(1-x_{i}\right)
$$

The detailed of encrypting image is described as follows:

Step 1. The key with 16 elements is randomly generated as the initial key and the initial key is implemented XOR operation with every pixel value of the plain image. The result of XOR operation is regard as the relating key; 
Step 2. According to initial condition of logistic maps, namely two parameters $\mu_{1}, \mu_{2}$ and two initial value $x_{1}^{1}(0), x_{1}^{2}(0)$, the relating key is evenly dividing relating key into four parts. These logistic maps are to iterate for 100 times to get rid of the transient effect of chaotic systems;

Step 3. The logistic maps are continuingly iterated base on the number of pixels, namely one map for the half number and the pseudorandom sequence consists of the logistic chaotic orbits;

Step 4. In order to permute the plain image, the chaotic orbits are sorted in ascending order. This operation (permutation) only changes the location of pixels of plain image;

Step 5. The XOR operation is implemented between the pixels of the permuted image and the pseudorandom sequence from the logistic maps. This operation (diffusion) only changes the value of pixels of digital image;

Step 6. According to the new DNA coding rule, the encrypted image is encoded by DNA coding;

Step 7. Outputting the encrypted image.

The flowchart of encrypting image is illustrated in Figure 1.

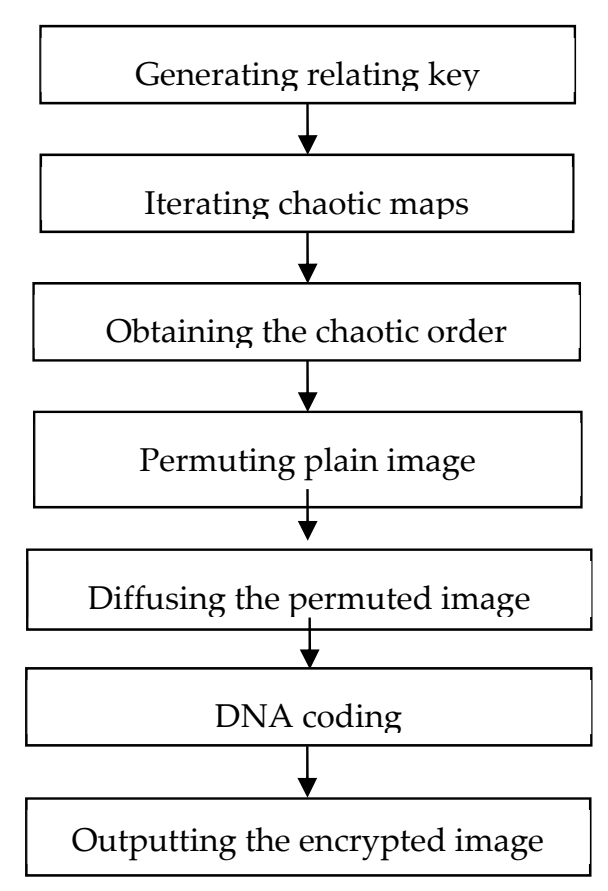

Figure 1. The flowchart of encrypting image.

\subsubsection{Decrypting Image}

The decryption process is similar to that of encryption procedure in the reversed order. It can be briefly stated as follows:

Step 1. According to the same relating key, the chaotic maps are to iterate for 100 times to get rid of the transient effect;

Step 2. The chaotic orbits are regenerated based on the same parameters and initial values as well as the encryption process;

Step 3. Decoding the cipher image based on the DNA coding rule;

Step 4. The XOR operation is implemented between the pixels of the cipher image and the pseudorandom sequence from the logistic maps and the permuted image is recovered;

Step 5. According to the order of chaotic sequences, the plain image is recovered from the permuted image;

Step 6. Outputting the plain image. 
Note that the permutation-diffusion architecture is widely used into image encryption based on chaotic map and DNA coding. So, the whole architecture of the proposed method is the permutation-diffusion. However, the previous works are mainly to convert the pixel value into an 8-bit binary sequence and then perform a simple one-to-one correspondence between the binary (or ASCII codes) and the DNA sequence without the function of error correction. For example, the binary sequence of the pixel value 1 is 00000001 and the corresponding DNA sequence is AAAC (A for 00, $\mathrm{C}$ for $01, \mathrm{G}$ for 10 and $\mathrm{T}$ for 11 ). In this paper, a DNA coding scheme with the function of error correction is proposed, where the pixel value of image is directly corresponded to a piece of DNA sequence with the function of error correction.

\section{Experiment and Simulation}

In order to resist the brute-force attack, the key space must be large enough for a secure image cryptosystem. 16 elements make up the key in our paper, key $=\left\{x_{i}\right\}, i=1,2, \ldots, 16, x_{i} \in[0,255]$. It is sufficiently large to ensure the security of digital image when the key space reaches to $2^{128} \approx 3.4 \times 10^{38}$. All the following experiment have the same size for key space.

\subsection{Key Sensitivity}

The test of key sensitivity can be stated as follows:

Step 1. Generating the key 123456789012345 and using this key to encrypt the test images;

Step 2. Generating another key-123456789012346 — with a slight difference and using this key to encrypt the same test image;

Step 3. Calculating the difference between different cipher images.

From the results, although the two different keys are only slightly different-by one bit-the cipher image with the key 123456789012345 is $99.63 \%$ different from the cipher image with the key 123456789012346. Figure 2 shows the results of test image Lena. For the same keys of Cameraman, there is $99.59 \%$ difference shown in Figure 3. There is $99.55 \%$ difference for Boat shown in Figure 4.

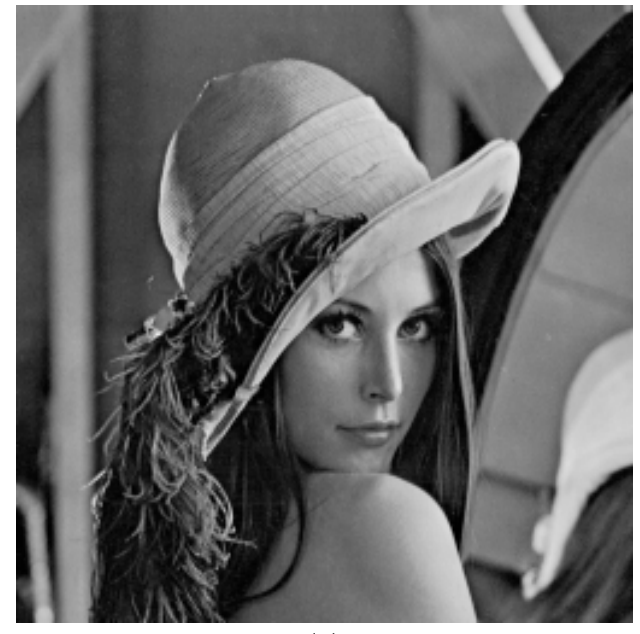

(a)

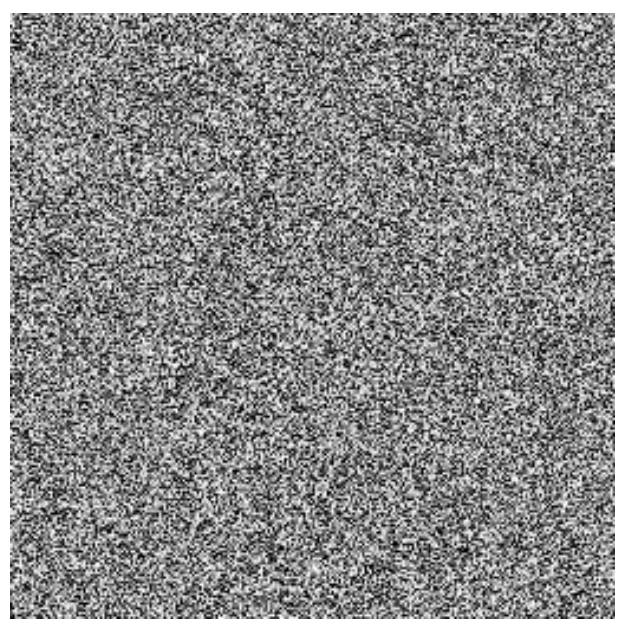

(b)

Figure 2. Cont. 


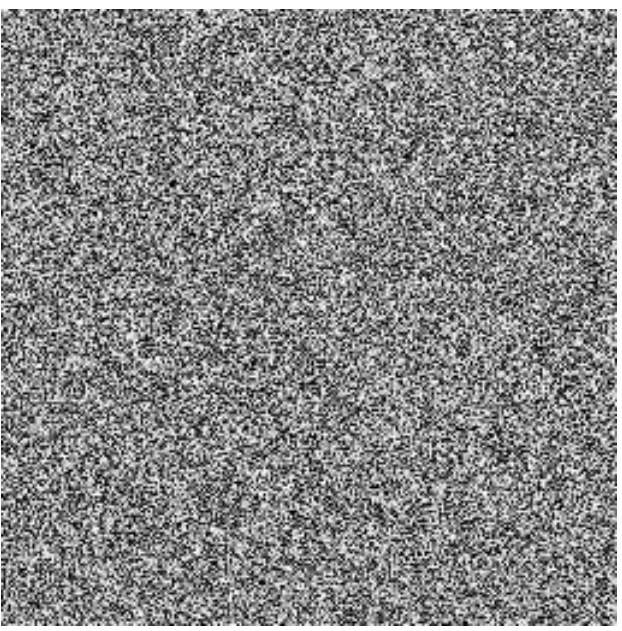

(c)

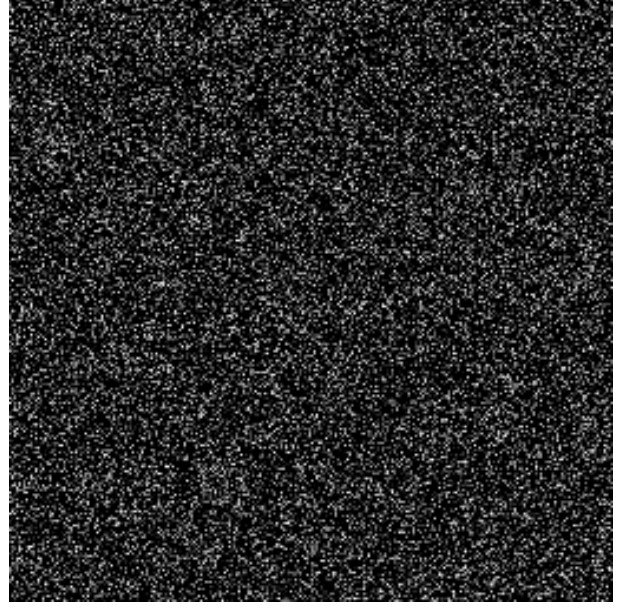

(d)

Figure 2. Key sensitivity for Lena. (a) Plain-image of Lena; (b) Encrypted image by key: 123456789012345; (c) Encrypted image by key: 123456789012346; (d) Difference image.

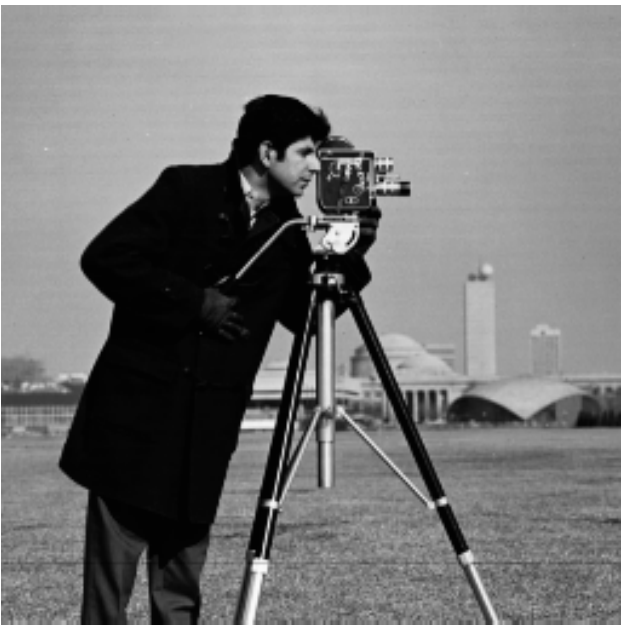

(a)

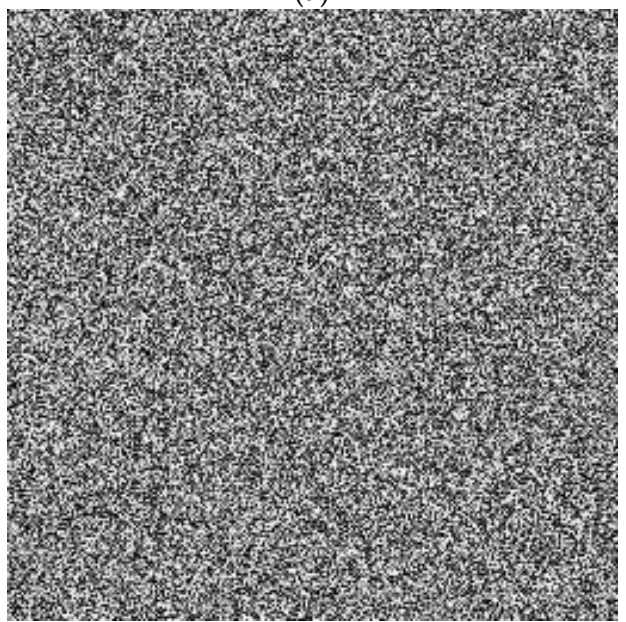

(c)

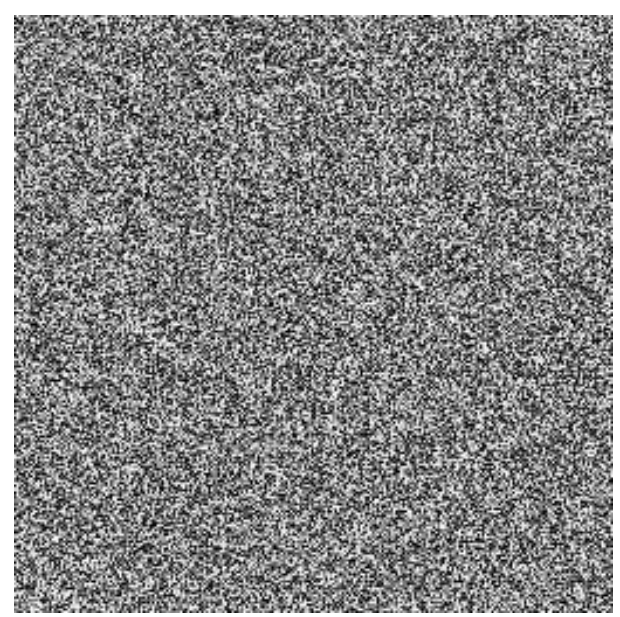

(b)

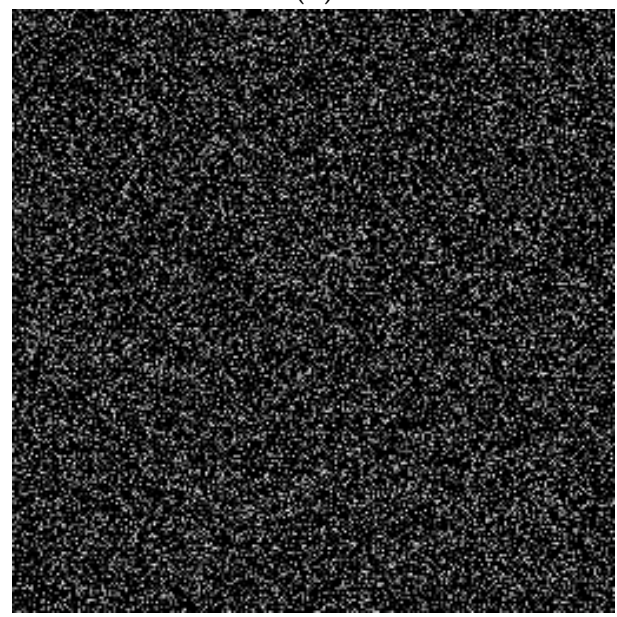

(d)

Figure 3. Key sensitivity for Cameraman. (a) Plain-image of Cameraman; (b) Encrypted image by key: 123456789012345; (c) Encrypted image by key: 123456789012346; (d) Difference image. 


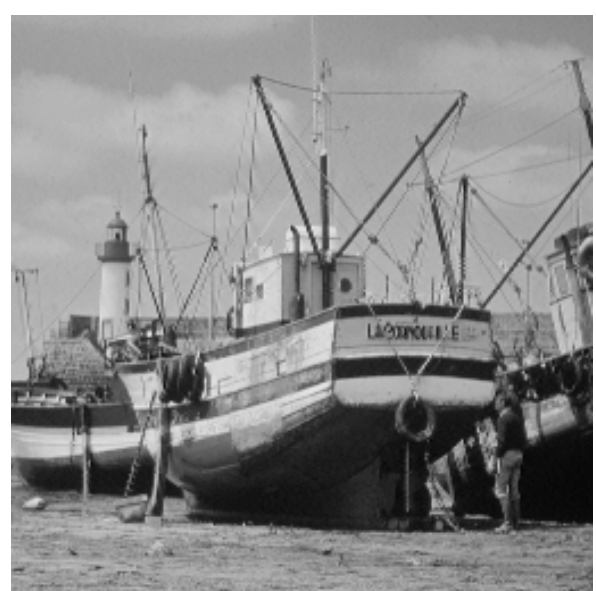

(a)

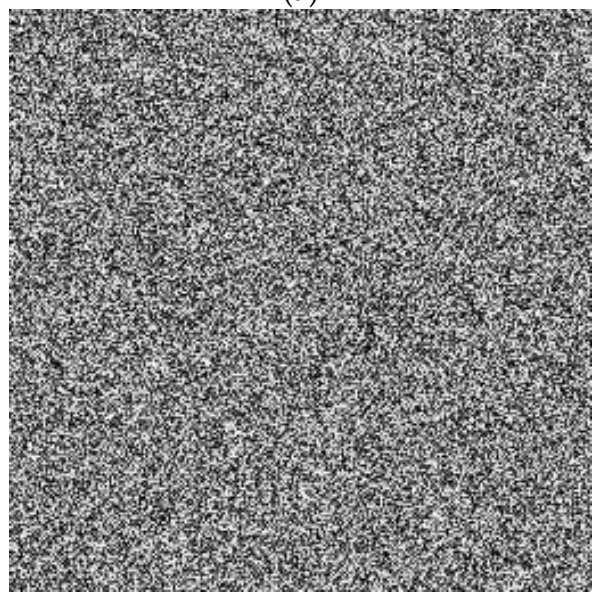

(c)

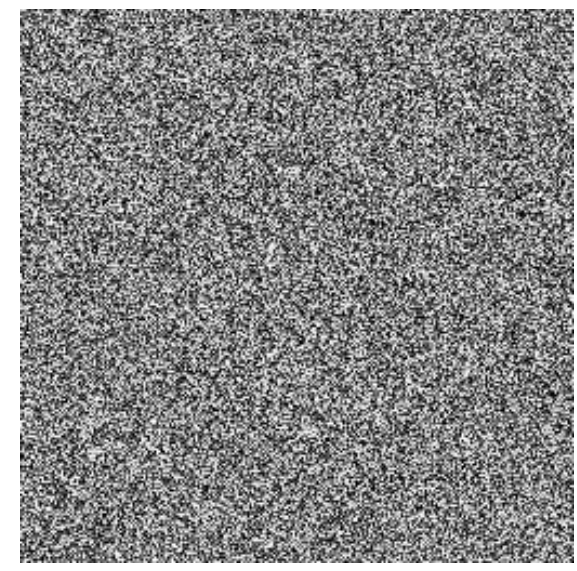

(b)

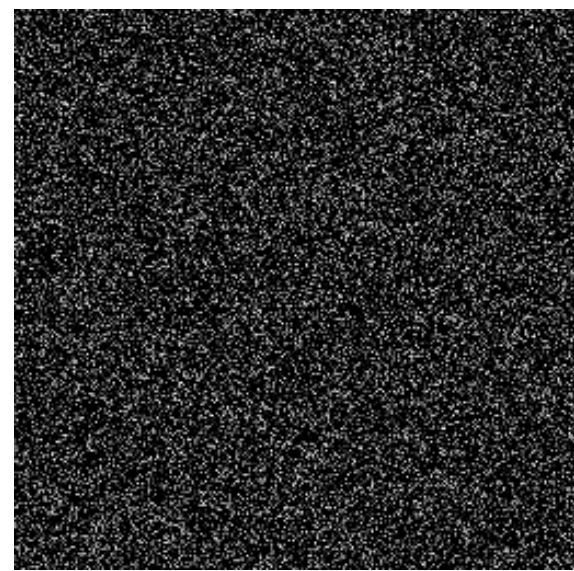

(d)

Figure 4. Key sensitivity for Boat. (a) Plain-image of Boat; (b) Encrypted image by key: 123456789012345; (c) Encrypted image by key: 123456789012346; (d) Difference image.

\subsection{Statistical Analysis}

The statistical characteristics of digital image can be exploited to attack the encryption system. The correlation of two adjacent pixels, as one of statistical characteristics of digital image, is the main aspect of statistical attack. 1000 pairs of adjacent pixels are respectively selected from vertical pixels, horizontal pixels and diagonal pixels. The correlation coefficient of each pair is calculated by the following formulas [1]:

$$
\begin{gathered}
\operatorname{cov}(x, y)=E\{(x-E(x))(y-E(y))\} \\
r_{x y}=\frac{\operatorname{cov}(x, y)}{\sqrt{D(x)} \sqrt{D(y)}}
\end{gathered}
$$

where $x$ and $y$ are grey-scale values of two adjacent pixels in the image. As digital image consists of discrete pixels, we adapt the following discrete formulas for calculating the correlation:

$$
\begin{gathered}
E(x)=\frac{1}{N} \sum_{i=1}^{N} x_{i} \\
D(x)=\frac{1}{N} \sum_{i=1}^{N}\left(x_{i}-E(x)\right)^{2}
\end{gathered}
$$




$$
\operatorname{cov}(x, y)=\frac{1}{N} \sum_{i=1}^{N}\left\{\left(x_{i}-E(x)\right)\left(y_{i}-E(y)\right)\right\}
$$

Table 3 shows the results of horizontal, vertical and diagonal directions. The values outside the brackets indicate the correlation between two adjacent pixels for three different plaintext images and the correlation between the cipher text images is indicated in the brackets. From the experimental results, the proposed algorithm greatly reduces the correlation between pixel values of horizontally, vertically and diagonally adjacent images and improves the ability to resist statistical attacks.

Table 3. The correlation coefficient of adjacent pixels.

\begin{tabular}{cccc}
\hline & Horizontal & Vertical & Diagonal \\
\hline Lena & $0.9727(0.0073)$ & $0.9481(0.0058)$ & $0.9250(-0.0091)$ \\
Cameraman & $0.9561(-0.0053)$ & $0.9213(-0.0062)$ & $0.9145(-0.0059)$ \\
Boat & $0.9334(0.0006)$ & $0.9249(0.0009)$ & $0.8891(-0.0002)$ \\
\hline
\end{tabular}

\subsection{Differential Attack}

Number of pixels change rate (NPCR) and Unified average changing intensity (UACI) are the common quantitative criteria for image cryptosystem to evaluate the property of resisting differential attack.

The NPCR and UACI are defined as follows [39-41]:

$$
\begin{gathered}
N P C R=\frac{\sum_{i, j} D(i, j)}{W \times H} \times 100 \% \\
U A C I=\frac{1}{W \times H}\left[\sum_{i, j} \frac{\left|C_{1}\left(i, j-C_{2}(i, j)\right)\right|}{255}\right] \times 100 \%
\end{gathered}
$$

where $C_{1}$ and $C_{2}$ denotes two different cipher images. These cipher images only have one pixel difference. $C_{1}(i, j)$ and $C_{2}(i, j)$ respectively denote the pixel values at the same point $(i, j)$ of $C_{1}$ and $C_{2}$; $H$ and $W$ are respectively the height and width of the image; $C_{1}(i, j)$ and $C_{2}(i, j)$ determine the value of $D(i, j)$, namely, if $C_{1}(i, j)=C_{2}(i, j)$ then $D(i, j)=0$ otherwise, $D(i, j)=1$.

The comparing results of NPCR and UACI list in the Table 4, where the image cryptosystem adapts the permutation - diffusion architecture with only one round.

The average of ten trials for our method is listed the table. According to the comparison, it shows that our method has higher security.

Table 4. The value of NPCR and UACI for Lena.

\begin{tabular}{lll}
\hline & NPCR & UACI \\
\hline Proposed algorithm & $99.57 \%$ & $32.38 \%$ \\
Wang's work [42] & $44.27 \%$ & $14.874 \%$ \\
Gupta's work [43] & $99.62 \%$ & $17.30 \%$ \\
\hline
\end{tabular}

\section{Correcting Errors}

In this chapter, we simulated the process of correcting errors. First, we encode the cipher image to DNA sequences and randomly change 1000 bases. Each DNA sequence encoded pixel only change one base. Figure 5 shows the effect of correcting errors. Figure 5 a shows the encrypted image contain 1000 errors. Figure $5 b$ shows the image after correcting errors by Hamming code. Figure $5 c$ shows the difference between Figure $5 \mathrm{a}$ and Figure $5 \mathrm{~b}$. Figure 5d shows the decrypted image after correcting 
errors. The experimental results express that the proposed method could effectively correct the errors and improve the accuracy of hybridization reaction.

Note that if the changed DNA sequence does not match the according to the DNA coding rule, we compulsively set this DNA sequence correspond to the pixel 255. For example, the pixel 16 match DNA sequence GCCTATCT according to DNA coding rule. If the third base is changed, namely GCGTATCT, there will be no pixel match this changed DNA sequence. So, we set GCGTATCT to correspond to pixel 255.

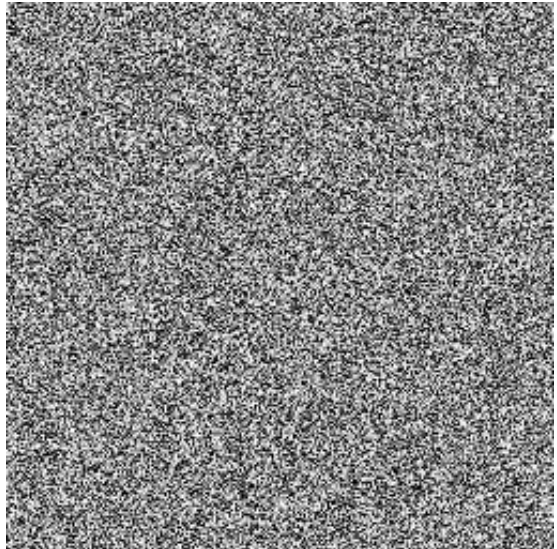

(a)

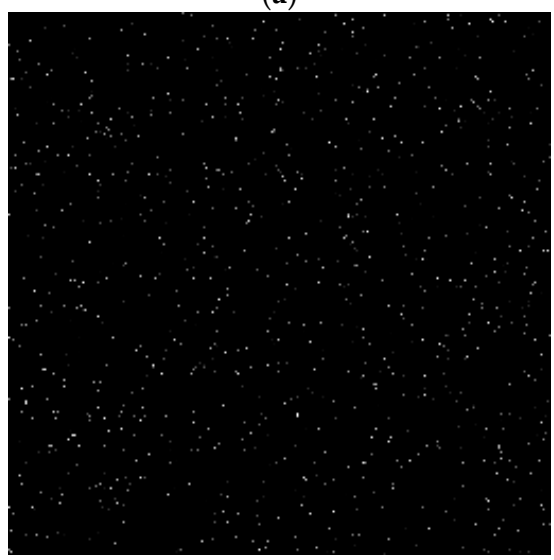

(c)

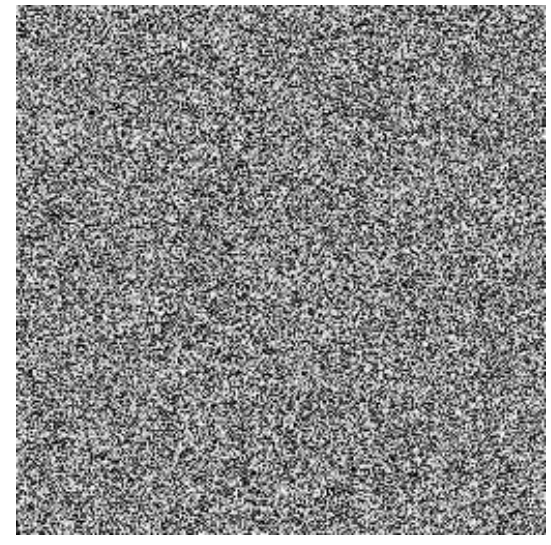

(b)

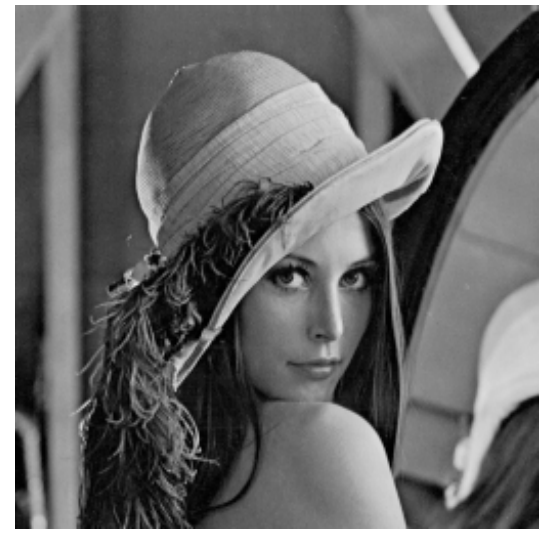

(d)

Figure 5. Correcting errors. (a) Containing errors image; (b) image after correcting errors; (c) Difference image; (d) Decrypted image after correcting errors.

\section{Conclusions}

In this paper, in order to improve the accuracy of DNA computing, we propose a novel method which could decrease the similarity of DNA sequences in DNA computing as well as correct errors from the process of image encryption and decryption. We first analyze the characteristic of DNA hybridization reaction and introduce the combinatorial constraints, namely Sequences-sequence Hamming Distance, Sequences-complementarity Hamming Distance and GC content, to design DNA coding. Then we use the chaotic map to generate pseudo-random sequences and encrypt the plain image by the permuting-diffusing architecture. Finally, we propose a novel DNA coding rule to encode the encrypted image. The experimental results show our method could be used to correct errors in image encryption based on DNA coding.

Bio-inspired computing models, such as membrane computing models [44-50], may provide intelligent methods for Image Encryption. As well, DNA coding strategies can provide biological ways in solving chemical information processing problems. 
Supplementary Materials: The supplementary materials are available online.

Author Contributions: Conceptualization, B.W.; Methodology, B.W.; Validation, B.W. and C.Z.; Formal Analysis, X.Z.; Investigation, Y.X.; Data Curation, S.Z.; Writing-Original Draft Preparation, B.W.; Writing-Review \& Editing, B.W.; Project Administration, B.W.; Funding Acquisition, B.W.

Funding: This work is supported by the National Natural Science Foundation of China (Nos. 61425002, 61751203, 61772100, 61702070, 61672121, 61572093), Program for Changjiang Scholars and Innovative Research Team in University (No. IRT_15R07), the Program for Liaoning Innovative Research Team in University (No. LT2015002), the Basic Research Program of the Key Lab in Liaoning Province Educational Department (No. LZ2015004).

Conflicts of Interest: The authors declare no conflict of interest.

\section{References}

1. Chen, G.; Mao, Y.; Chui, C.K. A symmetric image encryption scheme based on 3D chaotic cat maps. Chaos Solitons Fractals 2004, 21, 749-761. [CrossRef]

2. Wong, K.-W.; Kwok, B.S.-H.; Law, W.-S. A fast image encryption scheme based on chaotic standard map. Phys. Lett. A 2008, 372, 2645-2652. [CrossRef]

3. Lian, S.; Sun, J.; Wang, Z. A block cipher based on a suitable use of the chaotic standard map. Chaos Solitons Fractals 2005, 26, 117-129. [CrossRef]

4. Chang, W.L.; Guo, M.Y.; Ho, M.S.H. Fast parallel molecular algorithms for DNA-based computation: Factoring integers. IEEE Trans. Nanobiosci. 2005, 4, 149-163. [CrossRef]

5. Zhang, W.; Yu, H.; Zhao, Y.L.; Zhu, Z.L. Image encryption based on three-dimensional bit matrix permutation. Signal Process. 2016, 118, 36-50. [CrossRef]

6. Kulsoom, A.; Xiao, D.; Ur, R.A.; Abbas, S.A. An efficient and noise resistive selective image encryption scheme for gray images based on chaotic maps and DNA complementary rules. Multimedia Tools Appl. 2016, 75, 1-23. [CrossRef]

7. Zhang, Y.Q.; Wang, X.Y. A symmetric image encryption algorithm based on mixed linear-nonlinear coupled map lattice. Inf. Sci. 2014, 273, 329-351. [CrossRef]

8. Wang, X.Y.; Zhang, H.L. A novel image encryption algorithm based on genetic recombination and hyper-chaotic systems. Nonlinear Dyn. 2016, 83, 333-346. [CrossRef]

9. Zhang, Q.; Guo, L.; Wei, X.P. Image encryption using DNA addition combining with chaotic maps. Math. Comput. Model. 2010, 52, 2028-2035. [CrossRef]

10. Liu, H.J.; Wang, X.Y.; Kadir, A. Image encryption using DNA complementary rule and chaotic maps. Appl. Soft Comput. 2012, 12, 1457-1466. [CrossRef]

11. Wei, X.P.; Guo, L.; Zhang, Q.; Zhang, J.X.; Lian, S.G. A novel color image encryption algorithm based on DNA sequence operation and hyper-chaotic system. J. Syst. Softw. 2012, 85, 290-299. [CrossRef]

12. Babaei, M. A novel text and image encryption method based on chaos theory and DNA computing. Nat. Comput. 2013, 12, 101-107. [CrossRef]

13. Enayatifar, R.; Abdullah, A.H.; Isnin, I.F. Chaos-based image encryption using a hybrid genetic algorithm and a DNA sequence. Opt. Lasers Eng. 2014, 56, 83-93. [CrossRef]

14. Ozkaynak, F.; Yavuz, S. Analysis and improvement of a novel image fusion encryption algorithm based on DNA sequence operation and hyper-chaotic system. Nonlinear Dyn. 2014, 78, 1311-1320. [CrossRef]

15. Rehman, A.U.; Liao, X.F.; Kulsoom, A.; Abbas, S.A. Selective encryption for gray images based on chaos and DNA complementary rules. Multimedia Tools Appl. 2015, 74, 4655-4677. [CrossRef]

16. Song, C.Y.; Qiao, Y.L. A Novel Image Encryption Algorithm Based on DNA Encoding and Spatiotemporal Chaos. Entropy 2015, 17, 6954-6968. [CrossRef]

17. Wu, X.J.; Kan, H.B.; Kurths, J. A new color image encryption scheme based on DNA sequences and multiple improved 1D chaotic maps. Appl. Soft Comput. 2015, 37, 24-39. [CrossRef]

18. Wang, X.Y.; Zhu, X.Q.; Zhang, Y.Q. An Image Encryption Algorithm Based on Josephus Traversing and Mixed Chaotic Map. IEEE Access 2018, 6, 23733-23746. [CrossRef]

19. Parvaz, R.; Zarebnia, M. A combination chaotic system and application in color image encryption. Opt. Laser Technol. 2018, 101, 30-41. [CrossRef]

20. Adleman, L.M. Molecular computation of solutions to combinatorial problems. Science 1994, 266, 1021-1024. [CrossRef] [PubMed] 
21. Li, T.; Wang, E.K.; Dong, S.J. Potassium-Lead-Switched G-Quadruplexes: A New Class of DNA Logic Gates. J. Am. Chem. Soc. 2009, 131, 15082-15083. [CrossRef] [PubMed]

22. Qian, L.; Winfree, E.; Bruck, J. Neural network computation with DNA strand displacement cascades. Nature 2011, 475, 368-372. [CrossRef] [PubMed]

23. Scudellari, M. Inner Workings: DNA for data storage and computing. Proc. Natl. Acad. Sci. USA 2015, 112, 15771-15772. [CrossRef] [PubMed]

24. Wang, B.; Zhou, S.; Zheng, X.; Zhou, C.; Dong, J.; Zhao, L. Image watermarking using chaotic map and DNA coding. Opt.-Int. J. Light Electron Opt. 2015, 126, 4846-4851. [CrossRef]

25. Zhang, Q.; Wang, B.; Wei, X.; Fang, X.; Zhou, C. DNA word set design based on minimum free energy. IEEE Trans. NanoBiosci. 2010, 9, 273-277. [CrossRef] [PubMed]

26. Chai, X.L.; Chen, Y.R.; Broyde, L. A novel chaos-based image encryption algorithm using DNA sequence operations. Opt. Lasers Eng. 2017, 88, 197-213. [CrossRef]

27. Wu, X.J.; Kurths, J.; Kan, H.B. A robust and lossless DNA encryption scheme for color images. Multimedia Tools Appl. 2018, 77, 12349-12376. [CrossRef]

28. Marathe, A.; Condon, A.E.; Corn, R.M. On combinatorial DNA word design. J. Comput. Biol. 2001, 8, $201-219$. [CrossRef] [PubMed]

29. Shin, S.-Y.; Lee, I.-H.; Kim, D.; Zhang, B.-T. Multiobjective evolutionary optimization of DNA sequences for reliable DNA computing. IEEE Trans. Evol. Comput. 2005, 9, 143-158. [CrossRef]

30. Hamming, R.W. Error detecting and error correcting codes. Bell Syst. Tech. J. 1950, 29, 147-160. [CrossRef]

31. Zhang, Q.; Wang, B.; Wei, X.P.; Zhou, C.J. A Novel Constraint for Thermodynamically Designing DNA Sequences. PLoS ONE 2013, 8. [CrossRef] [PubMed]

32. Watson, J.D.; Crick, F.H.C. Molecular structure of nucleic acids-A structure for deoxyribose nucleic acid. Nature 1953, 171, 737-738. [CrossRef] [PubMed]

33. Zhang, Q.; Guo, L.; Wei, X.P. A novel image fusion encryption algorithm based on DNA sequence operation and hyper-chaotic system. Optik 2013, 124, 3596-3600. [CrossRef]

34. Kracht, D.; Schober, S. Insertion and deletion correcting DNA barcodes based on watermarks. BMC Bioinform. 2015, 16, 50. [CrossRef] [PubMed]

35. Wang, B.; Zhang, Q.; Zhang, R.; Xu, C.X. Improved Lower Bounds for DNA Coding. J. Comput. Theor. Nanosci. 2010, 7, 638-641. [CrossRef]

36. Su, X.; Li, W.; Hu, H. Cryptanalysis of a chaos-based image encryption scheme combining DNA coding and entropy. Multimedia Tools Appl. 2017, 76, 14021-14033. [CrossRef]

37. Zhang, Y. Breaking a RGB image encryption algorithm based on DNA encoding and chaos map. Int. J. Inf. Secur. 2014, 1, 22-28.

38. Liu, Y.; Tang, J.; Xie, T. Cryptanalyzing a RGB image encryption algorithm based on DNA encoding and chaos map. Opt. Laser Technol. 2014, 60, 111-115. [CrossRef]

39. Kwok, H.; Tang, W.K.S. A fast image encryption system based on chaotic maps with finite precision representation. Chaos Solitons Fractals 2007, 32, 1518-1529. [CrossRef]

40. Peng, J.; Zhang, D.; Liao, X. A digital image encryption algorithm based on hyper-chaotic cellular neural network. Fundam. Inf. 2009, 90, 269-282.

41. Wang, B.; Zheng, X.; Zhou, S.; Zhou, C.; Wei, X.; Zhang, Q.; Che, C. Encrypting the compressed image by chaotic map and arithmetic coding. Opt.-Int. J. Light Electron Opt. 2014, 125, 6117-6122. [CrossRef]

42. Wang, Y.; Wong, K.W.; Liao, X.; Chen, G. A new chaos-based fast image encryption algorithm. Appl. Soft Comput. 2011, 11, 514-522. [CrossRef]

43. Gupta, K.; Silakari, S. Novel Approach for fast Compressed Hybrid color image Cryptosystem. Adv. Eng. Softw. 2012, 49, 29-42. [CrossRef]

44. Yuan, S.; Deng, G.; Feng, Q.; Zheng, P.; Song, T. Multi-objective evolutionary algorithm based on decomposition for energy-aware scheduling in heterogeneous computing systems. J. Univ. Comput. Sci. 2017, 23, 636-651.

45. Wang, X.; Gong, F.; Zheng, P. On the computational power of spiking neural P systems with self-organization. Sci. Rep. 2016. [CrossRef] [PubMed]

46. Song, T.; Wong, P.Z.D.M.; Wang, X. Design of logic gates using spiking neural p systems with homogeneous neurons and astrocytes-like control. Inf. Sci. 2016, 372, 380-391. [CrossRef] 
47. Song, T.; Rodríguez-Patón, A.; Zheng, P.; Zeng, X. Spiking neural p systems with colored spikes. IEEE Trans. Cognit. Dev. Syst. 2018. [CrossRef]

48. Wang, X.; Zheng, P.; Ma, T.; Song, T. Computing with bacteria conjugation: Small universal systems. Moleculer 2018, 23, 1307. [CrossRef] [PubMed]

49. Zhang, L.; Yuan, S.; Feng, L.; Guo, B.; Qin, J.; Xu, B.; Lollar, C.; Sun, D.; Zhou, H. Pore-Environment Engineering with Multiple Metal Sites in Rare-Earth Porphyrinic Metal-Organic Frameworks. Angew. Chem. 2018. [CrossRef]

50. Zhang, M.; Xin, X.; Xiao, Z. A multi-aromatic hydrocarbon unit induced hydrophobic metal-organic framework for efficient C2/C1 hydrocarbon and oil/water separation. J. Mater. Chem. 2017, 5, 1168-1175. [CrossRef]

Sample Availability: Samples of the images and DNA sequences are available from the authors.

(C) 2018 by the authors. Licensee MDPI, Basel, Switzerland. This article is an open access article distributed under the terms and conditions of the Creative Commons Attribution (CC BY) license (http:/ / creativecommons.org/licenses/by/4.0/). 